\title{
Aircraft-based inversions quantify the importance of wetlands and livestock for Upper Midwest methane emissions
}

5

Xueying Yu, Dylan B. Millet, Kelley C. Wells, Daven K. Henze, Hansen Cao, Timothy J. Griffis, Eric A. Kort, Genevieve Plant, Malte J. Deventer, Randall K. Kolka, D. Tyler Roman, Kenneth J. Davis, Ankur R. Desai, Bianca C. Baier, Kathryn McKain, Alan C. Czarnetzki, A. Anthony Bloom

10

Correspondence to: Dylan B. Millet (dbm@umn.edu)

This file includes:

Figures S1 to S10

SI References 
a) ATOM3
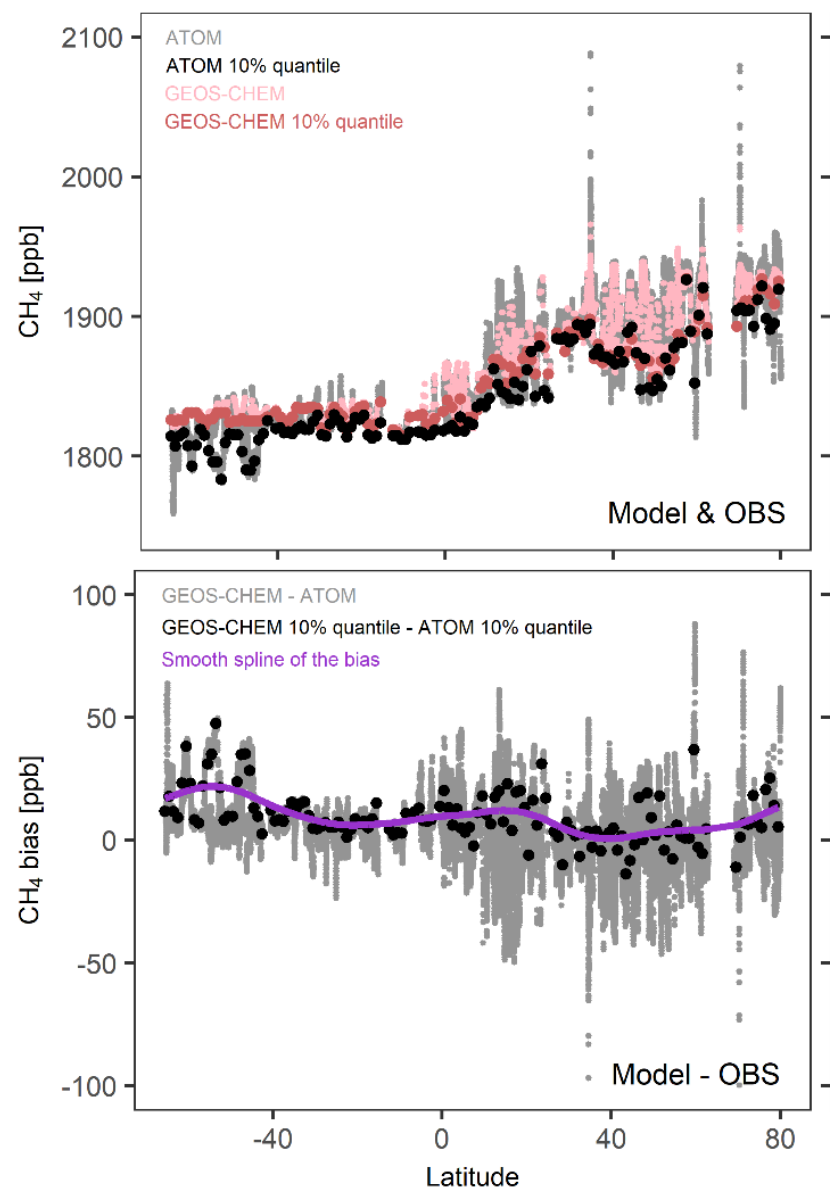

b) ATOM4
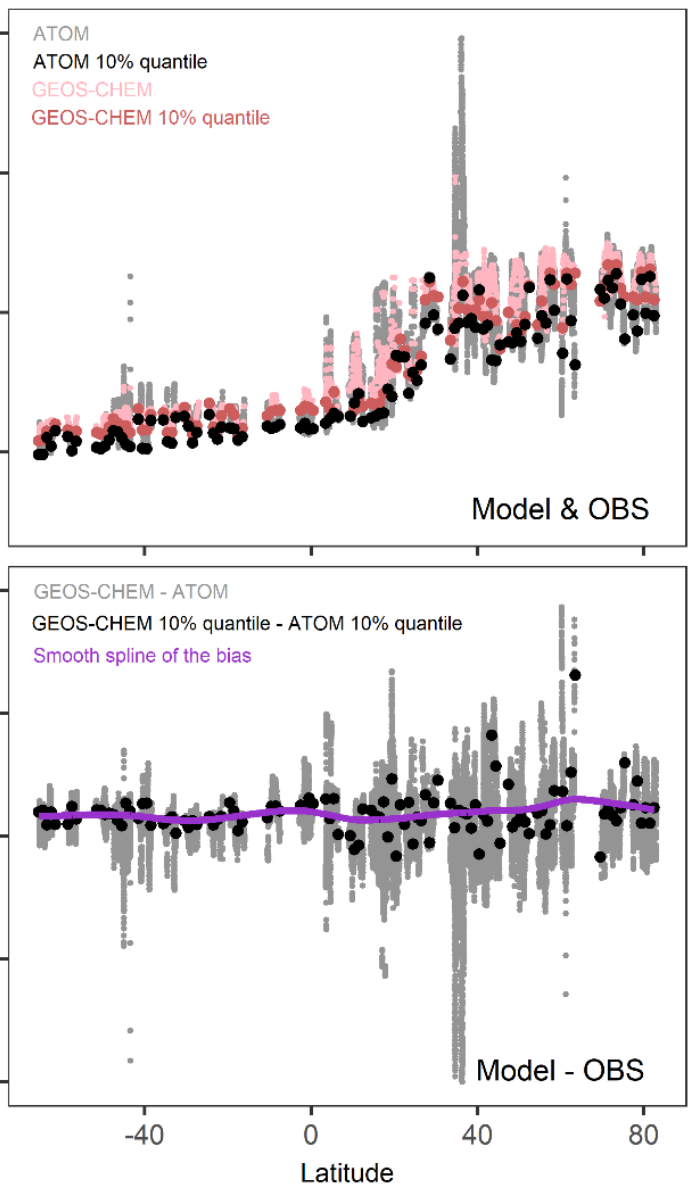

Figure S1. Correction of the model methane background based on aircraft measurements from ATom3 (left column) and ATom4 (right column). Plotted in the top panels are the mean (pink) and 0.1 quantile (red) observed tropospheric methane 20 mixing ratios by one-degree latitude bins, along with the corresponding model values (grey and black). Bottom panels show the resulting model-measurement mismatches with a smooth spline fit to the 0.1 quantile difference. 


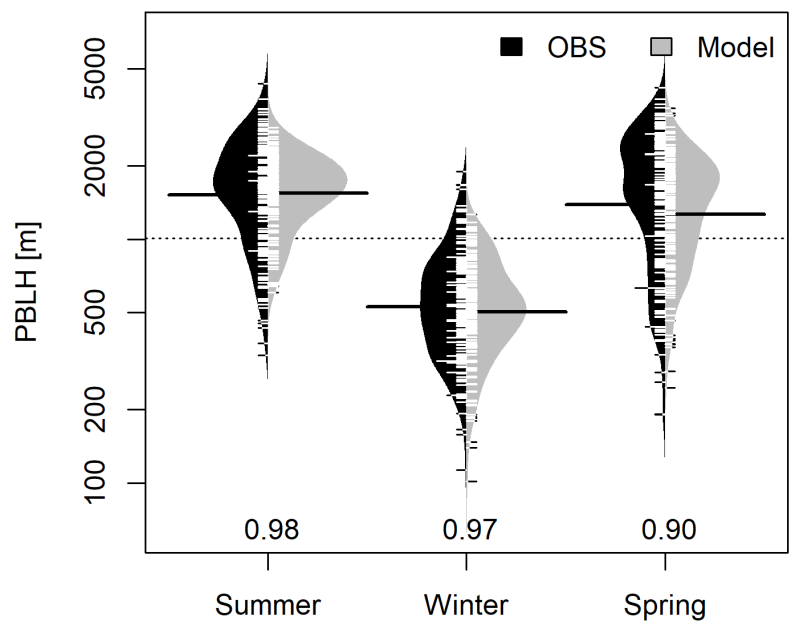

25 Figure S2. Model:measurement planetary boundary layer height (PBLH) comparison. The bean-plots compare radiosondebased (00:00 UTC, 18:00 or 19:00 local time) and GEOS-FP average midday (12:00-16:00 local time) PBLH values for summer (Aug. 2017, GEM1), winter (Jan. 2018, GEM2) and spring (May 2018, GEM3). Each bean displays density distributions for the radiosonde (black) and GEOS-FP (grey) data, along with the individual datapoints (short lines) and their mean values (black lines). The mean model:measurement ratios for each season are indicated above the X-axis. Comparisons include 168, 176, and 170 total data points in summer, winter, and spring respectively. 


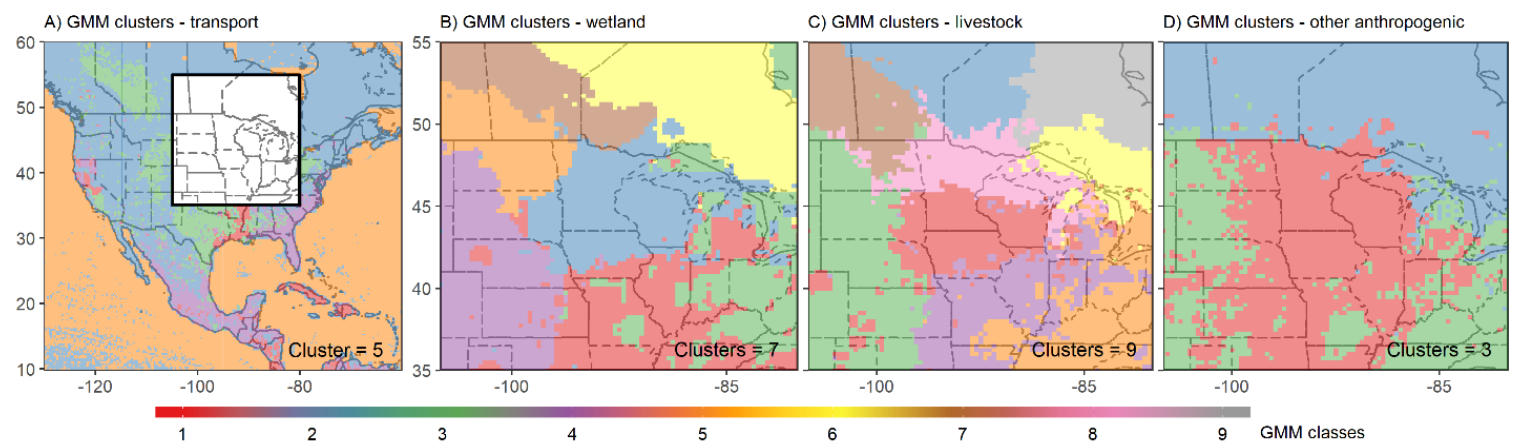

Figure S3. GMM clusters used for methane source optimization. As detailed in-text these include clusters representing transported emissions from outside the Upper Midwest domain (panel A), as well as Upper Midwest wetland (panel B),

35 livestock (panel C), and other anthropogenic (panel D) emissions. Not plotted are clusters (1 each) for Upper Midwest oil + gas + coal, rice, biomass burning, and other natural emissions. 


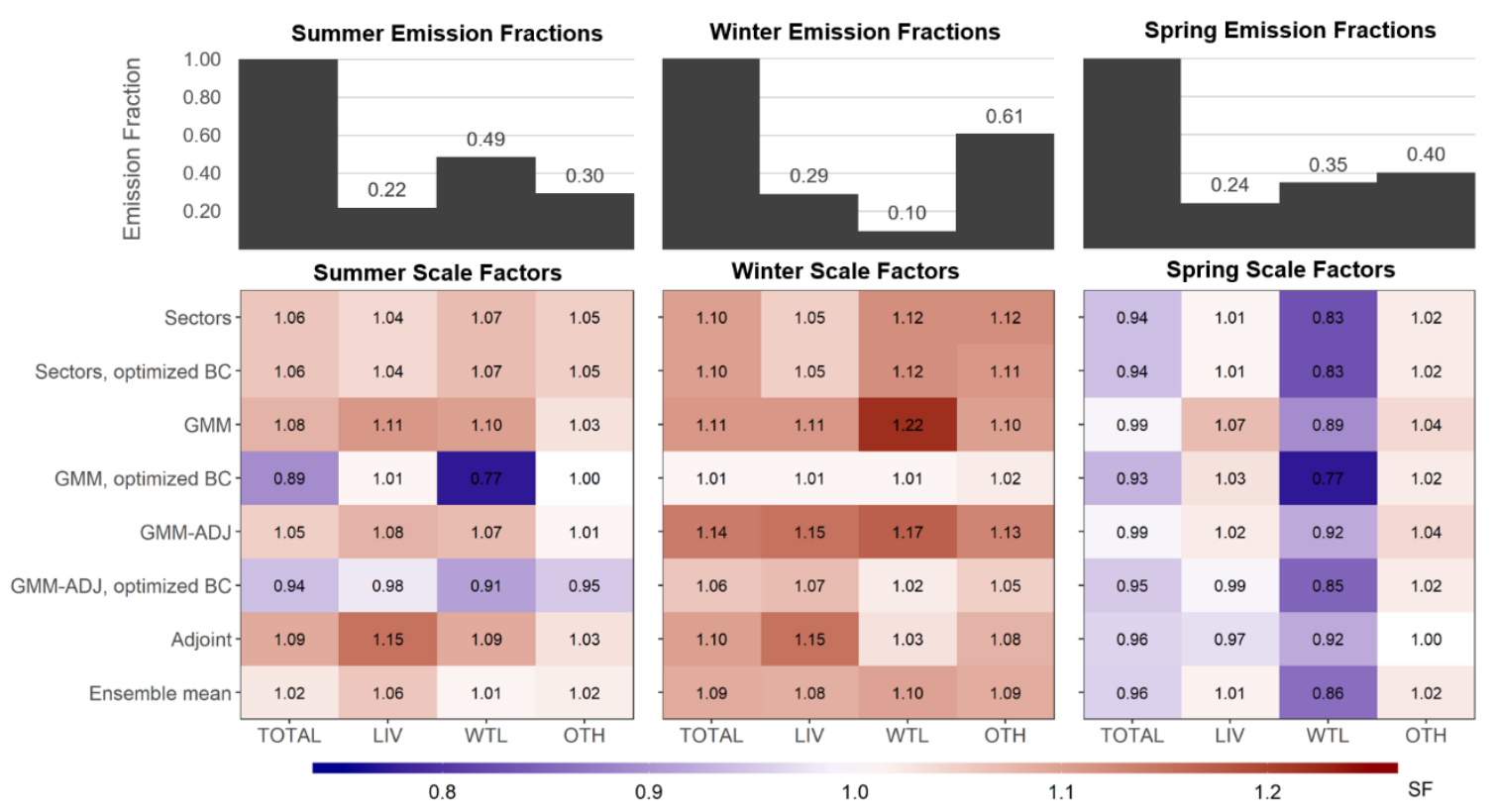

40 Figure S4. Same as Fig. 3 but using regulation parameter $\gamma=1$. 


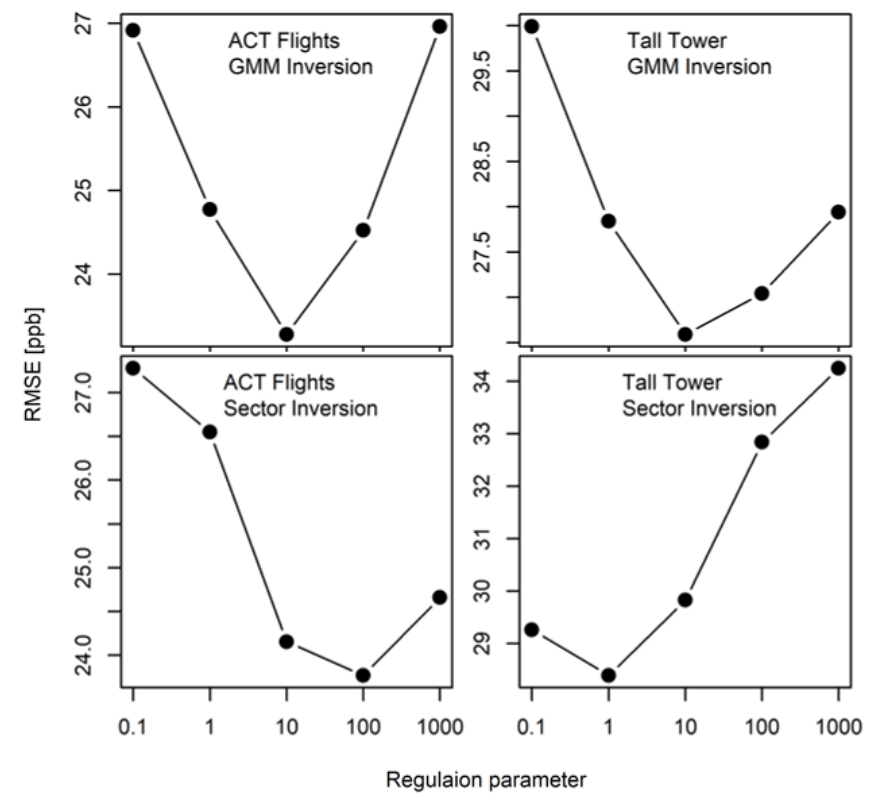

Figure S5. Aggregated level of agreement between optimized model results and independent measurements as a function of regularization parameter $(\gamma \in[0.1,1000])$. Plots show the root mean square error (RMSE) for the analytical (i.e., sector-

45 based and GMM) inversion results with respect to observations from the ACT-America campaign and the KCMP, LEF, and WSD tall towers. $\gamma=10$ is selected as the best case. 


\begin{tabular}{|c|c|c|c|c|c|c|c|c|c|c|c|c|c|c|c|c|}
\hline \multicolumn{5}{|c|}{ ACT flights } & \multicolumn{4}{|c|}{ WSD tower } & \multicolumn{4}{|c|}{ KCMP tower } & \multicolumn{4}{|c|}{ LEF tower } \\
\hline Prior- & 23.80 & 26.64 & 0.99 & 0.77 & .23 .22 & 23.54 & 0.99 & 0.72 & -59.71 & 69.30 & 0.97 & -0.10 & -5.13 & 28.73 & 1.00 & 0.07 \\
\hline Sectors - & -13.92 & 20.91 & 0.99 & 0.80 & -17.48 & 20.05 & 0.99 & 0.71 & -48.61 & 70.25 & 0.97 & -0.09 & -0.37 & 26.92 & 1.00 & 0.14 \\
\hline Sectors, optimized BC- & -13.85 & 20.88 & 0.99 & 0.80 & -15.89 & 18.49 & 0.99 & 0.74 & -44.63 & 66.75 & 0.98 & -0.02 & 3.96 & 27.43 & 1.00 & 0.23 \\
\hline GMM- & 7.61 & 18.62 & 1.00 & 0.77 & .11 .41 & 18.06 & 0.99 & 0.72 & .39 .42 & 66.79 & 0.98 & 0.04 & 2.56 & 23.28 & 1.00 & 0.36 \\
\hline GMM, optimized BC- & -0.67 & 18.48 & 1.00 & 0.77 & -3.04 & 14.80 & 1.00 & 0.75 & -35.37 & 64.63 & 0.98 & -0.06 & 6.26 & 21.90 & 1.00 & 0.36 \\
\hline GMM-ADJ - & -22.92 & 25.68 & 0.99 & 0.80 & -4.35 & 14.59 & 1.00 & 0.77 & -29.46 & 57.07 & 0.98 & 0.21 & 2.76 & 21.02 & 1.00 & 0.53 \\
\hline GMM-ADJ, optimized BC- & -19.93 & 24.12 & 0.99 & 0.79 & -15.37 & 18.74 & 0.99 & 0.73 & -52.19 & 65.81 & 0.97 & 0.08 & -13.68 & 20.65 & 0.99 & 0.44 \\
\hline Adjoint- & -9.66 & 19.93 & 0.99 & 0.78 & -8.21 & 16.40 & 1.00 & 0.76 & -32.53 & 56.03 & 0.98 & 0.34 & 4.86 & 17.43 & 1.00 & 0.63 \\
\hline Ensemble mean- & -12.65 & 20.10 & 0.99 & 0.80 & -10.82 & 16.33 & 0.99 & 0.75 & -40.31 & 63.68 & 0.98 & 0.06 & .0 .48 & 21.54 & 1.00 & 0.41 \\
\hline & M-O & $|\mathrm{M}-\mathrm{O}|$ & slope & $\dot{R}$ & $M-O$ & $|M-O|$ & slope & $\mathrm{R}$ & $M-O$ & $|\mathrm{M}-\mathrm{O}|$ & slope & $\dot{R}$ & $M-O$ & $|\mathrm{M}-\mathrm{O}|$ & slope & $\dot{\mathrm{R}}$ \\
\hline
\end{tabular}

Figure S6. Same as Fig. 4, but using independent measurements from the same timeframe as the GEM flights. See Sect. 2.4 50 for details. 
Apparent $\mathrm{q}_{10}$ of WetCHARTs ensemble mean

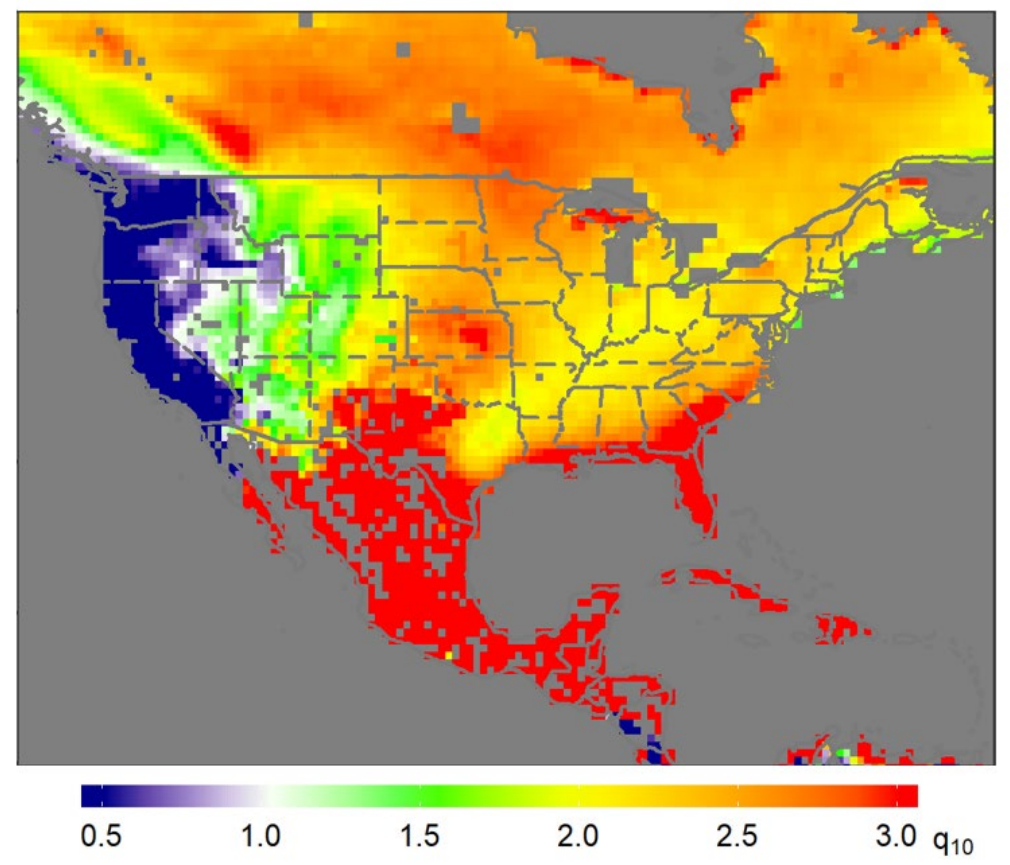

55 Figure S7. Net temperature dependence of methane emissions $\left(\mathrm{CH}_{4}: T q_{10}\right)$ for the WetCHARTs ensemble mean. Results are computed from the WetCHARTs methane emissions and surface skin temperatures from ERA-Interim (ECMWF, 2019). 


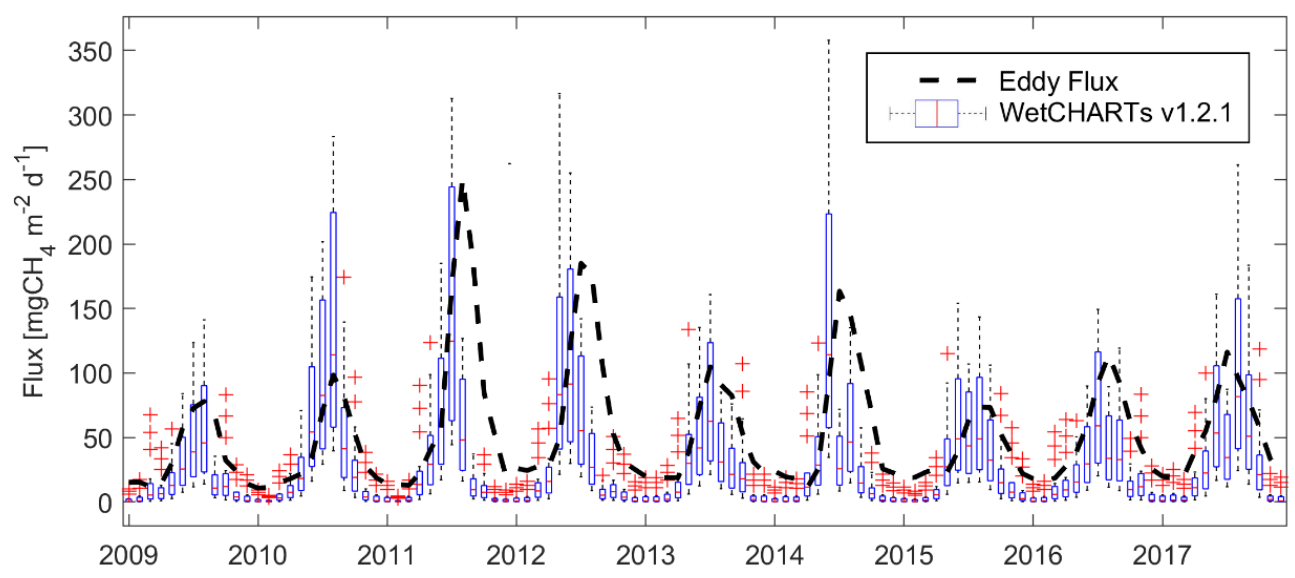

Figure S8. Monthly mean wetland fluxes at the Minnesota Bog Lake peatland during 2009-2017. Eddy covariance flux 60 measurements (black; (Deventer et al., 2019)) are compared to WetCharts predictions (blue). Boxes and whiskers encompass the interquartile range (IQR) and full range, respectively, across the WetCharts extended ensemble (excluding outliers). Outliers (defined as points exceeding the 0.25 or 0.75 quantile by $>1.5 \times$ the IQR) are plotted separately in red. 

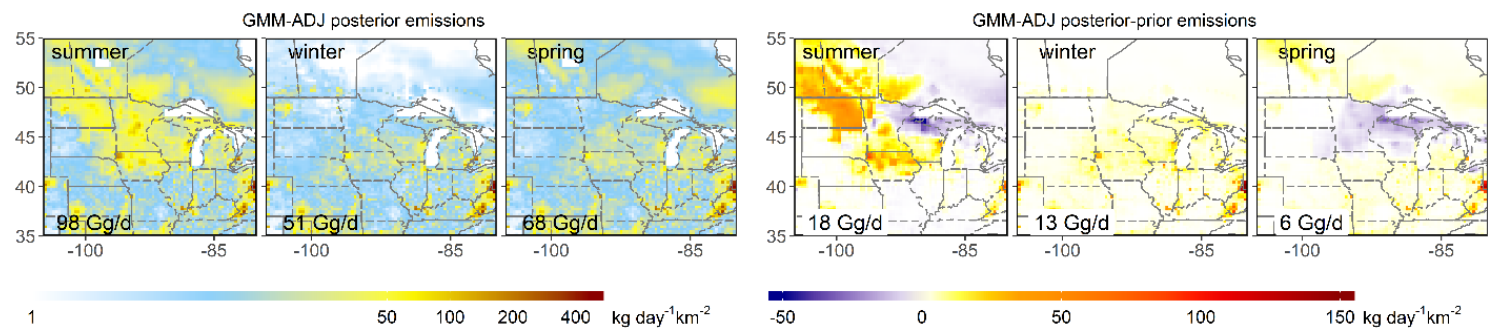

Figure S9. Same as Fig. 7, but showing results for the GMM-ADJ inversion. 

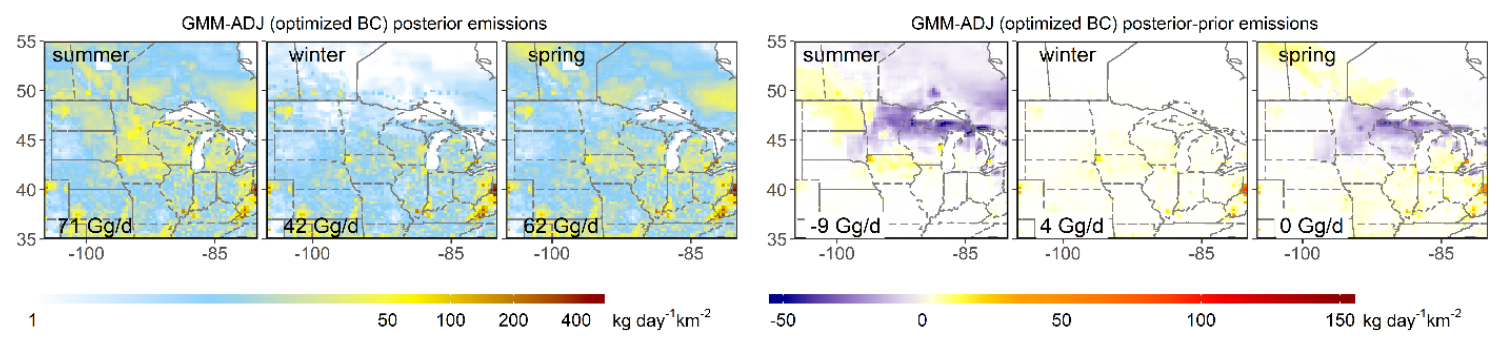

Figure S10. Same as Fig. 7, but showing results for the GMM-ADJ inversions with boundary condition optimization. 


\section{SI References}

Deventer, M. J., Griffis, T. J., Roman, D. T., Kolka, R. K., Wood, J. D., Erickson, M., Baker, J. M., and Millet, D. B.: Error characterization of methane fluxes and budgets derived from a long-term comparison of open- and closed-path eddy covariance systems, Agricultural and Forest Meteorology, 278, 107638, 10.1016/j.agrformet.2019.107638, 2019.

75 ECMWF: ERA-Interim, 2019. https://www.ecmwf.int. (access in June 2020). 